\title{
Lungosoprawivenza
}

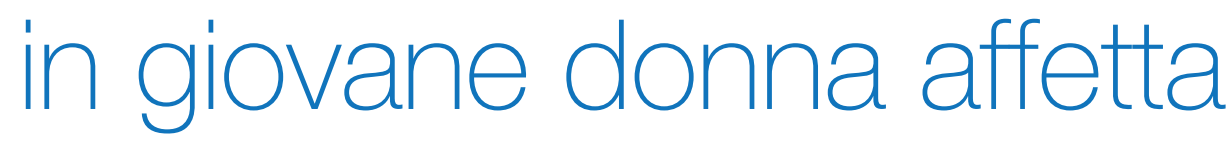

da tumore mammario

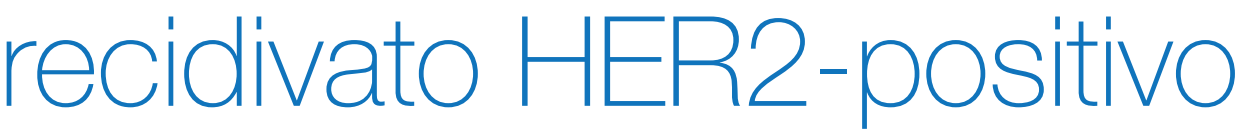

\section{Gianluca Cotroneo}

UOC Oncologia Medica, ASST Bergamo Est, Seriate (Bergamo)

\section{Abstract}

Si riporta il caso di una paziente con carcinoma mammario HER2-positivo, recidivata dopo 7 anni dalla diagnosi con recidiva tumorale HER2-positiva, trattata con farmaci a bersaglio molecolare anti-HER2 (pertuzumab, trastuzumab e lapatinib) associati a radioterapia e/o chemioterapia. Queste strategie terapeutiche hanno consentito un buon controllo della malattia che ha permesso alla paziente di proseguire in trattamento dopo circa 4 anni dalla recidiva.

\section{Situazione clinica iniziale e terapia adiuvante}

Alla visita senologica si apprezza adenopatia superfi-

\section{Indirizzo per la corrispondenza:}

Gianluca Cotroneo

e-mail: gianlucacotro@libero.it

Accettato: 31/12/2017 - Pubblicato online: 27/06/2018

C 2018 The Authors. This article is published by HPS Srl and licensed under Creative Commons Attribution-NC-ND 4.0 International (CC BY-NC-ND 4.0). Any commercial use is not permitted and is subject to Publisher's permissions. Full information is available at www.aboutpharma.com/publishing/riviste/aboutopen/ ciale al cavo ascellare sinistro delle dimensioni di circa una ciliegia, dura, fissa ai piani sottostanti e poco dolente motivo per cui si esegue lo stesso giorno un agoaspirato che risulta positivo. La paziente viene ricoverata presso il nostro reparto per il completamento della stadiazione e per una nuova biopsia escissionale della suddetta adenopatia allo scopo di effettuare una nuova valutazione immunoistochimica.

Durante la degenza esegue una tomografia a emissione di positroni (PET) total body con mezzo di contrasto (mdc) che mette in evidenza una captazione a livello linfonodale ascellare e sovraclaveare. Il reperto è confermato dalla tomografia assiale computerizzata (TAC) del torace che rileva tessuto neoformato con diametro trasverso di $4 \mathrm{~cm}$ a livello sottoclaveare sinistro e una lesione epatica di $2 \mathrm{~cm}$, risultata un angioma alla risonanza magnetica (RM) dell'addome. L'esame istologico della biopsia linfonodale eseguita depone invece per metastasi linfonodale da carcinoma duttale infiltrante con seguente immunoistochimica: ER/PgR negativi, Ki67 60\% e HER2 3+ (immunoistochimica variata rispetto al tumore primitivo del 2007). Si avvia quindi la paziente a un trattamento di I linea con doppio blocco (pertuzumab e trastuzumab) e docetaxel [1] per complessivi 9 cicli. La ri-stadiazione ogni 3 cicli documenta una malattia stabile secondo i criteri RECIST, fino a 
una risposta parziale agli ultimi 3 cicli con riduzione della colata adenopatica di oltre il 40\%. I marcatori tumorali CEA e Ca15.3 risultano nella norma.

Considerando la risposta ottenuta, si inizia una terapia di mantenimento con solo doppio blocco senza docetaxel, che la paziente esegue per un totale di 8 cicli. Le rivalutazioni ogni 3 mesi confermano la persistenza di risposta parziale fino a maggio 2016 quando il valore CEA risulta $58,1 \mathrm{ng} / \mathrm{ml}$, il Ca15.3 negativo e la PET evidenzia un significativo incremento di malattia in sede sovraclaveare sinistra (SUV max di 12 rispetto a 4.5 precedenti) con ipercaptazione maggiormente estesa in sede laterocervicale omolaterale. A completamento si esegue anche una colonscopia (per frequenti disturbi dell'alvo riferiti dalla paziente) risultata negativa.

Alla luce della progressione di malattia, sia strumentale che biochimica, e delle buone condizioni della paziente, si decide di avviare una II linea di terapia con lapatinib e capecitabina [2], che la paziente inizia a giugno 2016 ed effettua senza problematiche di tossicità, a dosaggio ottimale. La ri-stadiazione di malattia, eseguita dopo i primi 3 mesi, indica una risposta parziale di malattia con riduzione della captazione patologica dei linfonodi evidenziati alla PET e una risposta biochimica (CEA 12,8 ng/ml e Ca15.3 negativo). Considerata la buona tollerabilità al trattamento, si prosegue la terapia di II linea con il medesimo schema. Si eseguono in totale 9 cicli con ri-stadiazione ogni 3 mesi che indica una malattia stabile fino alla rivalutazione di marzo 2017, quando la PET rileva una progressione di malattia (seppur di modesta entità) delle note adenopatie sovraclaveari sinistre, comparsa di lieve captazione patologica dei linfonodi a livello mediastinico e nuovo aumento del CEA a 68 ng/ml (il Ca15.3 sempre nella norma). Si esegue una valutazione radioterapica che al momento esclude un trattamento locoregionale.

Alla luce della progressione strumentale e biochimica di malattia si decide di avviare la paziente a un trattamento di III linea con trastuzumab emtansine [3]. La paziente inizia tale terapia nel marzo 2017, eseguendo in totale 9 cicli di trattamento con ri-stadiazione intermedia ogni 3 cicli, che mette in evidenza una malattia stabile strumentale (il CEA presenta valori oscillanti da 68 fino a $82 \mathrm{ng} / \mathrm{ml}$ ) fino a ottobre 2017, quando la PET di stadiazione mostra una franca progressione a livello linfonodale ascellare, con tessuto neoformato di circa $6 \mathrm{~cm}$ infiltrante il plesso brachiale, che condiziona ipoestesia dell'arto superiore sinistro.

La paziente è pertanto avviata al trattamento radioterapico decompressivo con discreto beneficio e a novembre 2017 inizia una IV linea di terapia con re-challenge di trastuzumab e vinorelbina [4] di cui al momento ha eseguito 3 cicli di trattamento, e alla prima rivalutazione di malattia del marzo 2018 mostra una risposta biochimica completa (CEA e Ca15.3 negativi).

\section{Considerazioni cliniche}

L'iperespressione HER2 rappresenta circa il 20\% dei tumori metastatici della mammella ed è associata a un decorso clinico aggressivo. La possibilità di utilizzare farmaci a bersaglio molecolare che vanno ad agire sul recettore HER2 ha permesso di migliorare la sopravvivenza in questo setting di pazienti. Nell'ambito della sequenza chemioterapica da utilizzare va considerato il meccanismo d'azione di questi farmaci; infatti mentre trastuzumab, pertuzumab e trastuzumab emtansine legano la porzione extracellulare del recettore HER2 (anche se in diversi siti), lapatinib viene internalizzato nella cellula e si intercala nel sito di legame per l'ATP, non solo di HER2 ma anche di HER1, agendo quindi anche sugli eterodimeri HER1-HER2 [5]. Mentre i primi tre anticorpi monoclonali per ottenere il massimo di risposta necessitano che sulla superficie della cellula tumorale vi sia una elevata espressione della proteina HER2 (potendo comunque avere difficoltà nel riconoscere le forme di HER2 modificate o addirittura tronche), lapatinib agisce all'interno della cellula ed è in grado di agire sulle forme modificate o tronche della proteina HER2, addirittura favorendone un'iperespressione sulla superficie cellulare e quindi un'eventuale nuova ri-sensibilizzazione a trastuzumab. 


\section{Disclosures}

Questa pubblicazione è stata resa possibile grazie a un grant non condizionante di Istituto Gentili.

Il dott. G. Cotroneo dichiara di non avere conflitti di interesse in relazione all'articolo pubblicato.

\section{Bibliografia}

1. Baselga J, Cortés J, Kim S et al.; CLEOPATRA Study Group. Pertuzumab plus trastuzumab plus docetaxel for metastatic breast cancer. N Engl J Med 2012;366:109-19.

2. Cameron D, Casey M, Oliva C et al. Lapatinib plus capecitabine in women with HER-2 positive advanced breast cancer: final survival analysis of a phase III randomized trial. Oncologist 2010;15(9):924-34

3. Verma S, Miles D, Gianni L et al.; EMILIA Study Group. Trastuzum ab emtansine for HER2-positive advanced breast cancer. N Engl J Med 2012 Nov 8;367(19):1783-91.

4. Chan A. A review of the use of trastuzumab (Herceptin) plus vinorelbine in metastatic breast cancer. Ann Onco 2007;18(7):1152-8

5. Scaltriti M, Verma C, Guzman M et al. Lapatinib a HER2 tyrosine kinase inhibitor induces stabilization and accumulation of HER2 and potentiates trastuzumab-dependent cell cytotoxicity. Oncogene 2009;28:803-14. 